\title{
"Space Frames": Using space, color and animation in the visualization of complex graph systems
}

\author{
Elif Ayiter \\ Sabanci University \\ Istanbul, Turkey \\ aviter@sabanciuniv.edu \\ Ismail Kasarci \\ Sabanci University \\ Istanbul, Turkey \\ kasarci@sabanciuniv.edu
}

\author{
Selim Balcisoy \\ Sabanci University \\ Istanbul, Turkey \\ balcisoy@sabanciuniv.edu \\ Uraz C. Turker \\ Sabanci University \\ Istanbul, Turkey \\ urazc@su.sabanciuniv.edu
}

\author{
Murat Germen \\ Sabanci University \\ Istanbul, Turkey \\ muratgermen@gmail.com
Merve Cayli
Sabanci University
Istanbul, Turkey \\ mervec@su.sabanciuniv.edu
}

\begin{abstract}
This study concerns the development of $3 D$ interfaces that aid the navigation of large data structures through the usage of the HSV color space, and the ensuing "spatial frames". While data objects that have been colorized in various hues that carry equal saturation and brightness/transparency value seem to be equally fore grounded; varying saturation, brightness and transparency values add depth and hierarchy. During our experiments we noticed that, when thus colorized, the edges connecting the nodes form visual spatial frames, which can result in the meaningful partitioning of $3 D$ space. This property can be exploited to facilitate the display of overall trends within data sets, as well as to ease navigation.
\end{abstract}

\section{Project Description}

We have implemented a three-layered graph layout, generated from spherical coordinates. The position of each node in the layout is computed according to the number of links it has with other nodes. The nodes that have a significantly higher number of connections than the average number of links are identified as hubs. The system also allows users to modify three parameters of the positioning algorithm depending on the data: global radius, cluster radius, and layer distance, finding the most suitable representation to accomplish tasks such as searching for a critical path between clusters.

In a three dimensional graph, the design space is enlarged considerably and factors such as form, lighting and viewing angles become critical considerations. Furthermore in $3 \mathrm{D}$, objects take on a quality of concreteness that facilitates remembrance and recognition as well as placement in the context of other objects. Further reports regarding the usage of 3D space in info-vis have been given by Einsfeld, et al. [01] and Franck and Ware [02]. Experiments involving a 3D graph tracing task showed that subjects were able to comprehend about three times as much information in an interactive, 3D environment as in a comparable $2 \mathrm{D}$ one

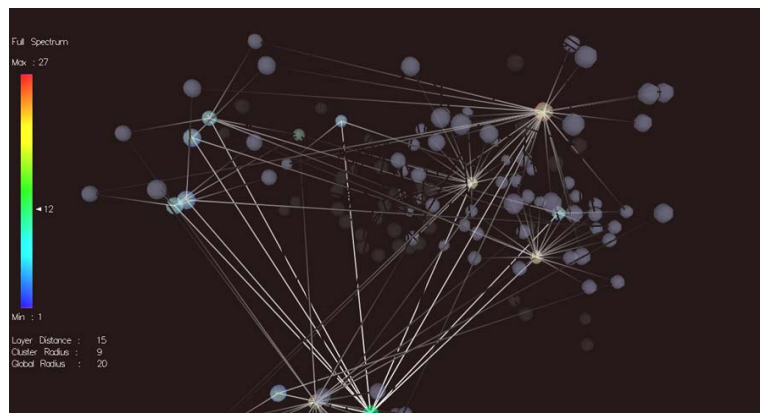

While data objects that have been colorized in various hues that carry strong saturation and brightness value seem to be equally fore grounded; varying values add depth and hierarchy: Less saturated, darker values recede into the background, while brighter, saturated objects tend to become fore grounded. Yet another benefit of using the full HSV space is the utilization of color in the creation of "meaningful landmarks" [03]. Brighter, nodes and connecting paths, in juxtaposition to their darker counterparts tend to attain landmark attributes with much greater facility than those in systems where these values are ignored.

In their inactive, i.e. deselected state, all nodes are represented in shades of grey, which can still be clearly distinguished form the charcoal grey background and also have differentiated lightness values, depending 
upon their rate of connectivity. Nodes with low connectivity are in darker tones, receding into the background, whereas hubs are in lighter values. In their inactive state all connecting paths are grey. When hubs are selected they, and their first and second order connections, become colorized according to the color scheme with which the diagram is viewed. In comparison to their inactive state, the connecting paths are also emphasized by lighter tones of grey, which progressively darken the further apart from the hub the connection occurs. While the examined hub and its connections are fore grounded the overall diagram is still visible, but back grounded.

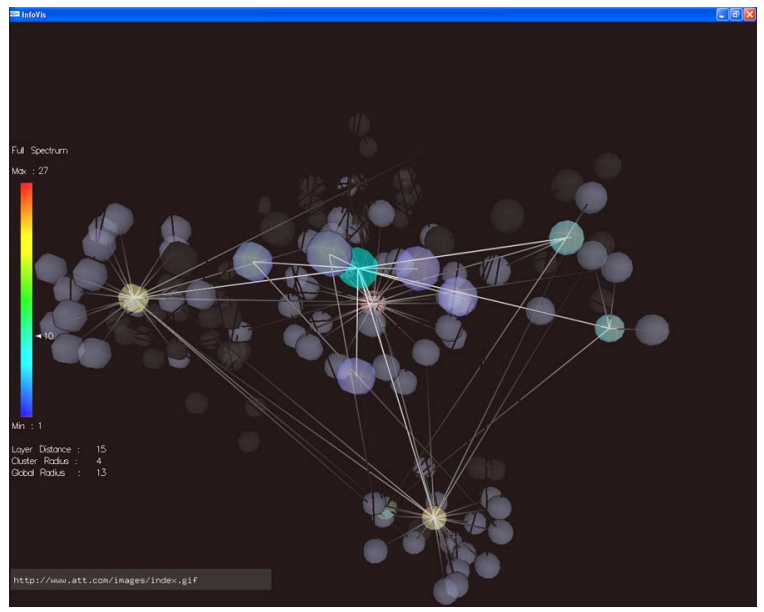

Monochromatic, analogous, triadic and complementary contrast color schemes were used. The first two of these are in accordance with Tufte [04], who proposes harmonies; while the latter follows Itten [05] on the usage of color contrasts. To compute the hue color of a node we first normalized its weight and multiplied the normalized value with the spectral range. After the system assigns the color of a node, we then applied a saturation algorithm. According to a hub's depth value, descendent nodes were rendered as less saturated. Edges that define path between nodes are also rendered in same manner. The saturation algorithm alters the value of a node depending on its depth value. Thus, first order descendents of a selected hub will be brighter than the second order descendents but darker then the selected hub. We also saw that when color is applied adroitly to the connecting paths, these create "spatial frames", which partition the homogenous 3D space, provided that they are fore grounded sufficiently from the rest of the construct. Since the system increases the transparency of the unselected nodes and edges, thus backgrounding them, the illumination of the selected paths re-defined the concept of linkage, creating a dramatic virtual architecture. This property can then be exploited to display overall trends and create meaningful space partitioning, easing user navigation we focused exclusively on the connecting paths in the second phase of investigations. Animations where these connections and hierarchies manifest themselves as progressively transparent paths starting from the selected node were also found to facilitate the display of overall trends structure.

\section{Results and Discussions}

While usage of the HSV color space aids the detection of connections between data, not all data sets can be examined under the same color scheme. While data with well connected hubs will benefit from the implementation of a complementary or analogous scheme, data that is comprised of numerous nodes, such as was the case with our sample data set, will benefit from the implementation of a fuller spectrum.

In most visual/spatial systems landmarks can be discerned by viewers, either as geographic, ornamental or focal elements. The homogeneous and abstract nature of graphs, however, makes the differentiation of data objects, to the extent where they might indeed acquire landmark attributes a considerable challenge. Our present study in the usage of the HSV color space in 3D graphs has provided us with valuable clues in how to proceed with the task at hand using color.

\section{References}

[01] Einsfeld, K,, Agne, S., Deller, M., Ebert, A., Klein, B., Reuschling, C. Dynamic Visualization and Navigation of Semantic Virtual Environments. Proceedings of the Information Visualization (IV’06), IEEE. 2006

[02] Franck, G., Ware, C., Representing Nodes and Arcs in 3D Networks Proceedinggs. IEEE Conference on Visual Languages. St. Louis, 1994. 189-190.

[03] Chen, C. Information Visualization: Beyond the Horizon. Springer, London. 2004. P: 22, 287.

[04] Tufte, E. R. Envisioning information. Graphic Press, Connecticut, 1990.

[05] Itten, J. The Art of Color. John Wiley and Sons. 2000. 